\title{
Use of video technology to enhance telemedicine applications
}

\begin{abstract}
Telemedicine uses information and communication technologies (ICT) to deliver healthcare services remotely, thus removing geographical barriers. Video technology can effectively facilitate the growth of telemedicine. This paper discusses the applications of video technology to enhance telemedicine services. The general functions of telemedicine videoconferencing systems are reviewed. The display technology is also discussed. It is expected that advanced monitors with $8 \mathrm{~K}$ resolution can be used in future telemedicine videoconferencing systems to significantly improve the image quality and service performance.
\end{abstract}

Keywords: video technology, telemedicine, blood pressure, temperature, healthcare professionals, monitors
Volume 7 Issue 2 - 2021

\author{
Jian-Guo Zhang \\ Department of Electrical and Electronic Engineering, London \\ South Bank University, UK
}

Correspondence: Jian-Guo Zhang, Department of Electrical and Electronic Engineering, London South Bank University, I03 Borough Road, London, SEI OAA, UK,

Email zhangj@Isbu.ac.uk, jian-guo-zhan@126.com

Received: February 22, 2021 | Published: March 09, 2021
Abbreviations: ICT, information and communication technologies; UHD, ultrahigh-definition; CRT, cathode-ray tube; LED, light-emitting diode; LCD, liquid-crystal display; CCFL, cold cathode fluorescent lamp

\section{Introduction}

Advances in electronics and digital technology have facilitated to lower down the costs of wearable and connected medical devices, smartphones (including 5G mobile phones), computers equipped with ultrahigh-definition (UHD) monitors, videoconferencing and medical information systems, while improving their functionality. This in turn can provide new challenge and opportunity for using information and communication technologies (ICT) to meet an increasing demand for high-quality healthcare services in a costeffective manner. Nowadays, the widespread use of wearable devices and smartphones with embedded sensors makes it possible to continuously monitor users' personal health and to track their daily activities, for example, recording blood pressure, temperature, sleep patterns, heart rate, electrocardiogram, oxygen saturation, and respiration rate. ${ }^{1,2}$ In doing so, users can take control of their own health. This also enables to remotely collect users' real-time health data and could even allow to send patient's health information to a doctor or other healthcare professional over the internet for fast diagnosis if health-related issues are detected. ${ }^{1}$ It in turn can avoid time passing and developing symptoms for which patients may want to seek medical advice from doctors at a later stage rather than being aware of health concern or problem in real time to reduce the risk. The use of smartphones, wearable devices, computers and fast Internet substantially facilitates telemedicine applications. Telemedicine is described by the World Health Organization as the delivery of healthcare services at a distance by all healthcare professionals using ICT to exchange valid information for diagnosis, treatment and prevention of disease and injuries, research and evaluation, and for the continuing education of healthcare providers. ${ }^{3}$ This in turn enables to improve clinical management and delivery of healthcare services worldwide by means of the increased access, quality, efficiency, and cost-effectiveness. Telemedicine is particularly useful for people with reduced mobility or who live in rural and remote areas with few health services and staff. As a result, the distance and time barriers between healthcare providers and patients can be effectively overcome. Then telemedicine enables to connect rural and remote sites with healthcare professionals around the world. ${ }^{3}$ Telemedicine applications have grown significantly in recent years. Recent progress in ICT has led to a radical transformation in healthcare industry.

\section{Telemedicine}

Telemedicine uses ICT to provide healthcare remotely when the participants are physically separated, especially for patients with limited access to healthcare services. ${ }^{3-5}$ This is done by telephone consultation, videoconferencing and email, respectively. The relevant information can be, for example, text messaging, audio, video, and still images which can be transmitted over high-speed optical fiber and wireless communication networks (including $5 \mathrm{G}$ mobile networks). Telemedicine applications can be synchronous or asynchronous/storeand-forward, depending on the timing of the information transmitted and the interaction between the individuals involved (e.g., health professional-to-health professional or health professional-to-patient). ${ }^{3}$ Real time or synchronous telemedicine requires the involved individuals to be simultaneously present for immediate exchange of information, as in the case of videoconferencing with a clinician. On the other hand, asynchronous/store-and-forward telemedicine may encompass review of pre-recorded data such as clinical information through an electronic consultation, or of pathology and/or radiology images, etc. ${ }^{3,6}$ In these cases, the transmitted information can be text, audio, video, or still images. Telemedicine delivers secure virtual healthcare and allows doctors to visit their patients in a virtual manner. In doing so, it is typically billed at a lower cost than inperson visits and allows to provide affordable healthcare services for more people to access. ${ }^{7}$ Moreover, patients using telemedicine do not need to travel to medical centers, take time off work, or spend time waiting for providers. ${ }^{4}$ Therefore, remote care and diagnosis offered by telemedicine can benefit patients and healthcare systems with the reduction of travel costs for specialist care and the associated time and stress when compared to the case of in-person visits. ${ }^{3}$ Then significant cost-savings to patients and healthcare systems can be achieved, ${ }^{4}$ and 
health outcomes are improved. Furthermore, the use of telemedicine can avoid costly hospitalizations and can help patients in healthcare through remote patient monitoring and incorporating patients and family members into the care process, ${ }^{5}$ This can allow patients to seek earlier treatment and improve the quality of life for patients who have chronic conditions.

By delivering healthcare remotely, in-person care can be focused in the hospital to only those patients who really need it. This in turn allows to scale healthcare systems, protect lives, and safeguard economic activity. ${ }^{8}$ It is also possible that telemedicine will transform the delivery of healthcare services by migrating such delivery away from hospitals and clinics into homes. ${ }^{3}$ Telemedicine applications not only can improve healthcare systems, but also create an interconnected global health network responsive to humanitarian crises. ${ }^{5}$ At present, telemedicine continues to grow, with the latest technology driving costs down and making the process affordable to more healthcare providers and their patients. ${ }^{9}$ The COVID-19 pandemic has affected healthcare systems globally. Due to the requirements for social distancing and quarantining, virtual visits have been demanded in order to avoid overloading the healthcare system with normal cases which can be handled without in-person visits to the hospital. As a result, telemedicine has been rapidly adopted to evaluate and treat patients. ${ }^{4,7,8}$ Telemedicine is a major driver in shifting the healthcare delivery model.
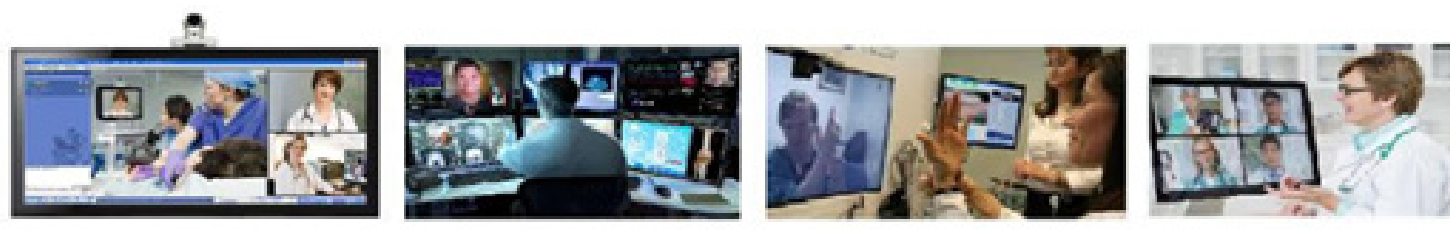

Figure I Examples of telemedicine videoconferencing (from websites).

The use of two-way video communications for telemedicine can effectively reduce travel costs and time in waiting rooms, improve patient care outcomes. This in turn can reduce overall healthcare costs, while keeping the face-to-face online interactions which are useful for patients. Telemedicine delivered over secure cloud-based video conferencing services will help shape the future of healthcare industry. ${ }^{11}$ In doing so, health information will be automatically captured using telemedicine devices (e.g., apps and sensors). As a result, patients will be able to continue to self-monitor their health and to send the measured results electronically. Then doctors will be able to react to the received data with no need of always seeing patients during office visits. Instead, patients will be seen using video communications from the comfort of their homes at the available time. ${ }^{11}$ Videoconferencing systems use video and communication technologies to transmit medical information such as audio, video, and graphics between two or more sites. Therefore, the main components of a telemedicine videoconferencing system generally include a codec, viewing monitor(s), camera(s), control/user interaction devices (e.g., mouse, keyboard), input devices (e.g., document scanner, medical scopes), and output and storage devices (e.g., printers, CD-ROM drives, USB). ${ }^{10}$ It is expected that video conferencing can speed up a shift of telemedicine into homes.

\section{Display technology}

From the above discussions on videoconferencing systems, we can see that high-performance displays are very important for videoconferencing applications (Figure 2). To make confident

\section{Videoconferencing systems for telemedicine}

Clearly, telemedicine services rely on ICT to deliver healthcare services and to transmit health information. One of its main supporting technologies is videoconferencing. As discussed in the above, real time uninterrupted video streaming is required by synchronous telemedicine. Since healthcare professionals and patients are connected via live interactive video, this in turn can enhance patient-provider communications. Video technology can effectively facilitate the growth of telemedicine. At present, virtual visits are more important than in the past due to the COVID-19 pandemic and physical distancing requirements. Video conferencing enables healthcare providers to set their own schedules and maintain patient contact with less travel and less pressure to treat as many patients as possible in a set time. ${ }^{9}$ This can include new-patient visit, follow-up visit, and on-treatment visit, respectively. Videoconferencing systems can be used for diagnosis and prescription of medical treatment for patients at remote locations, for remote clinical consultations between medical professionals, and for education and training of medical staff, etc (Figure 1). Telemedicine can be a simple telephone conversation between personnel or email exchange, or a real-time interactive video examination of a patient conducted by physicians remotely. ${ }^{10}$ For example, doctors can use video technology to quickly diagnose a stroke victim's symptoms and suggest immediate action to minimize further damage to the brain, or bring in specialists around the country to provide instant care. ${ }^{9}$ diagnoses and to improve detection, the high resolution of a colour display is required to show high-quality or perfect images over time. This can, for example, help radiologists in checking the smallest clinical details in order to make confident diagnoses and to improve detection. In doing so, the radiologists are able to detect suspicious findings in the earliest stage. ${ }^{12}$ Display technology has evolved from early cathode-ray tube (CRT) monitors to plasma display panels, and recently, to flat-panel displays using liquid-crystal display (LCD) or light-emitting diode (LED) technology. These were primarily utilized for TV and computer applications in the past. Moreover, recent advances in display technology have enabled new form factors for mobile and wearable displays, such as flexible and transparent displays, to support novel applications. CRTs are a vacuum glass tube with electron gun(s) and a phosphor-coated screen, which have a dependence of tube length on the screen size and normally occupy a considerable amount of desk space. Unfortunately, consumer preference for larger screen sizes implies that the tube length of CRTs would have to be increased correspondingly. In doing so, CRTs would become very bulky and heavy. ${ }^{14}$ As a result, flat-panel displays with light weight and space saving have replaced CRTs. For example, energy-efficient, cost-effective LCD screens are widely used in today's TV sets, computers, and various handheld devices. Plasma display panels are also a type of flat-panel displays and were already used for large-screen HDTV application. A plasma display panel is comprised of millions of tiny cells which are sandwiched between two glass panels sealed around the edges and are filled with a mixture of xenon and neon gases. ${ }^{14}$ When the voltages representing a video signal are applied, the electrodes are charged to excite a gas mixture, 
thus changing the gas into a plasma state. Then the ionized gas emits ultraviolet light which in turn excites the red, green and blue phosphors coated inside three separate sub-pixel cells, respectively, to emit the colored light of the pixel. A plasma display has the drawbacks of relatively high power consumption and phosphor burnin, ${ }^{15}$ respectively. A declining demand for plasma display panels has resulted in discontinuing plasma TV production in the consumer market. LCDs are a flat-panel display with low power consumption and cost. Liquid crystals do not emit light, but their electro-optic effect is exploited to function like a light shutter for controlling the passage of light. This implies that the external light is required by a LCD to display the information through an appropriate illumination scheme. ${ }^{15} \mathrm{~A}$ LCD generally has two main components: ${ }^{13,16}$

a. A liquid crystal of which the cells act as a light shutter to block or allow the external light to pass through a series of optical filters in order to produce images according to an applied voltage;

b. A backlight unit for illumination of a LCD panel.

Previously, backlights used a cold cathode fluorescent lamp (CCFL) as the light source. However, LEDs have recently replaced CCFLs as a light source for backlighting, because the former has advantages of lower power consumption, longer lifetime, and smaller dimensions over the latter. To implement backlights, light sources can be either distributed evenly over the area of the display or placed along one or more edges of a light guide that spreads light over the display area. In effect, the thinnest LCDs were designed by using the LED edge-lit scheme. ${ }^{13}$ Usually, LCD TVs with LED backlights are called the LEDbacklit LCD TVs (or LED LCD TVs) or simply referred to as LED TVs. Organic LED displays are a kind of emissive display technology, which are currently used in digital cameras, smartwatches, high-end TVs, flagship smartphones, and new concept cars, respectively. An OLED consists of thin layers of organic material sandwiched between two electrodes which are deposited on a glass or plastic substrate. ${ }^{15,17}$ When an electric current flows through the OLED, the visible light is thus generated by its light-emitting layer. ${ }^{18}$ Ultrahigh-definition TVs with $8 \mathrm{~K}$ resolution $(7680 \times 4320$ pixels $)$ are commercially available now, which use LCD and OLED displays, respectively (Figure 3 \& Figure 4). Since high-performance $8 \mathrm{~K}$ displays are able to show lifelike images and to ensure an immersive viewing experience closer to reality, they can be used in telemedicine videoconferencing systems to significantly improve the quality of healthcare services. Sharp company already reported to deliver the world's first $8 \mathrm{~K}$ image monitor in November 2017 to the medical field with an 8K rigid endoscope system. By transmitting UHD and realistic images in real time to remote areas and by viewing on a large screen $8 \mathrm{~K}$ image monitor, this can be applied to telemedicine and medical education. The recorded $8 \mathrm{~K}$ surgical video of a leading surgeon can be also used for education of young doctors (Figure 5). ${ }^{19}$
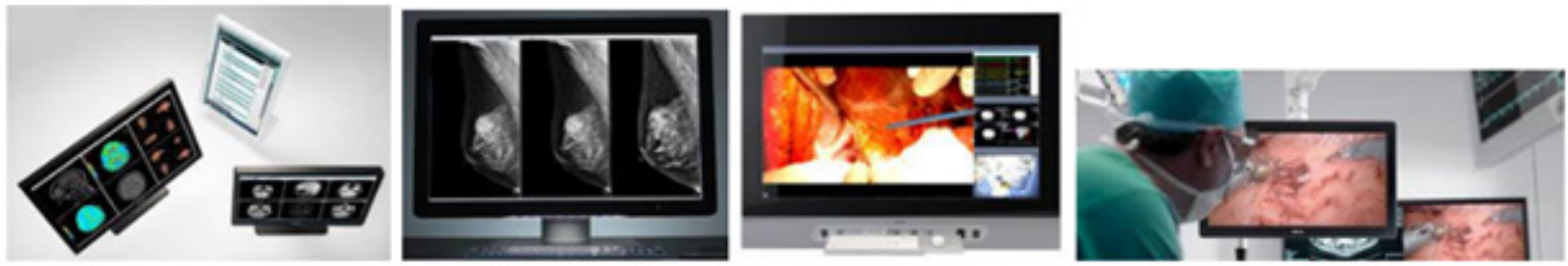

Figure 2 Barco's medical displays. ${ }^{12}$

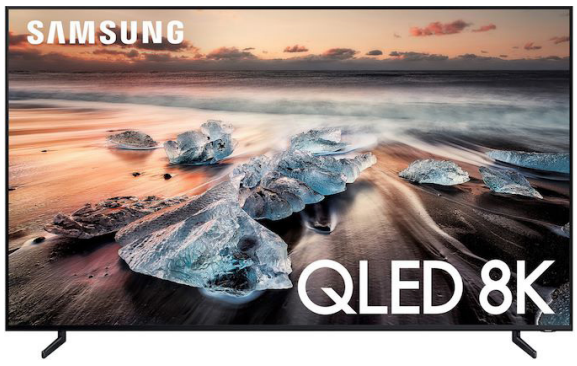

A

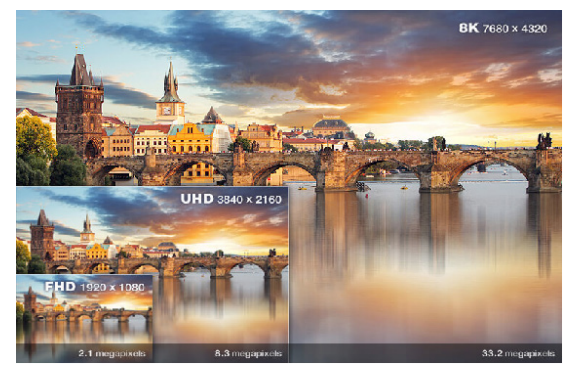

C

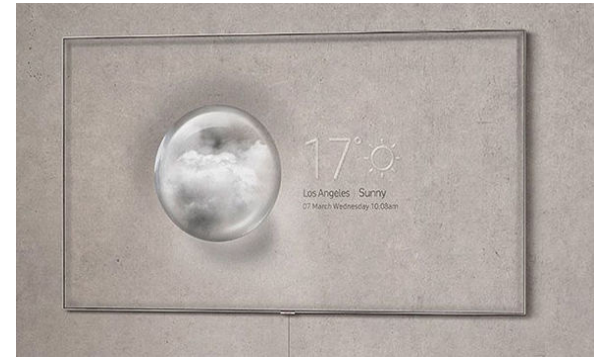

B

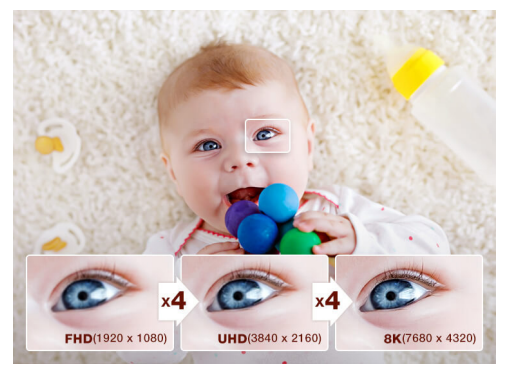

D

Figure 3 Samsung's TV. (A) 8K QLED UHDTV using quantum dot enhanced LCD. (B) QLEDTV with Ambient Mode. (C) Illustration of full high-definition (FHD), $4 \mathrm{~K}$ and $8 \mathrm{~K}$ resolutions for TV screens. (D) Comparison of $4 \mathrm{~K}$ and $8 \mathrm{~K}$ images with FHD image on TV screens (a larger resolution creates a sharper image, giving the viewer greater detail. The increase of resolutions improves the image quality when looking closely at the same visuals. https://pid.samsungdisplay.com/en/ learning-center/blog/8k-resolution-advancements). Note that Ambient Mode is a feature of Samsung QLED TVs. Ambient Mode allows a TV to show the same pattern as the wall behind it so that the TV is almost invisible and to display photos and images on the TV. It can also show useful information such as time and weather. 

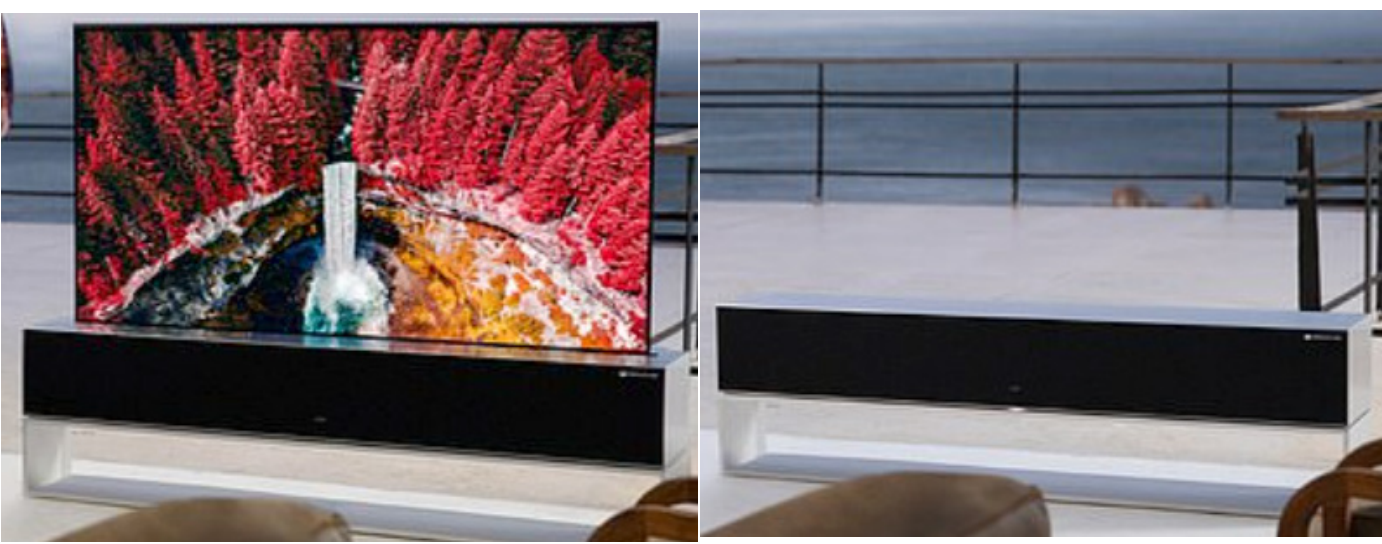

Figure 4 LG's rollable 65-inch TV with an OLED 8K screen. (A) The screen is unrolled. (B) The screen is rolled up in the aluminium base when not in use. https:// www.dailymail.co.uk/sciencetech/article-8858399/
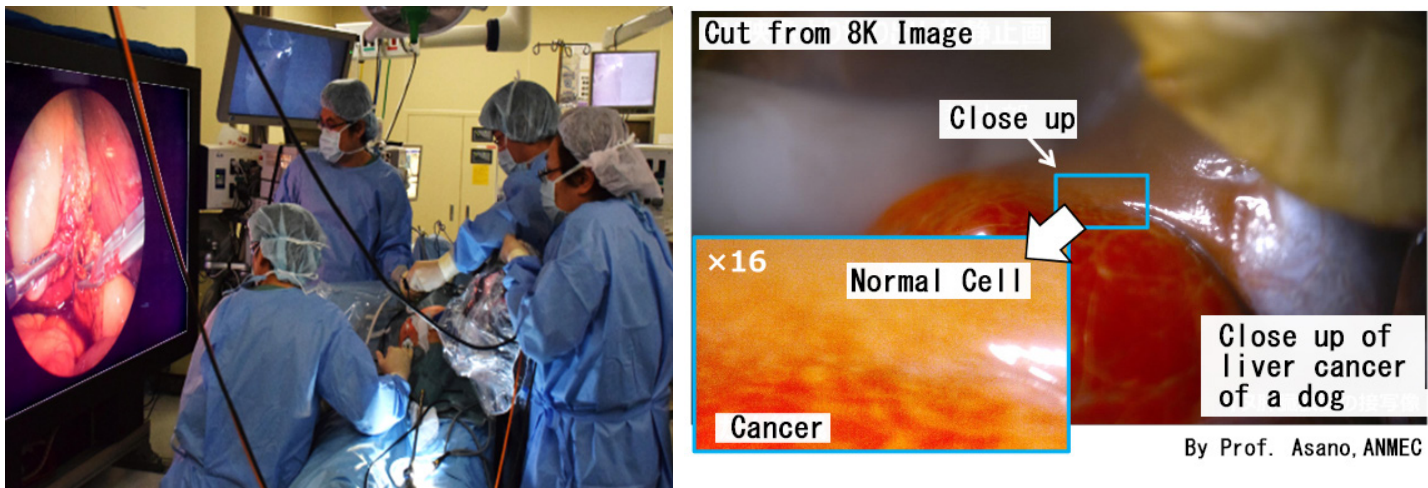

Cancer

By Prof. Asano, ANMEC

Figure 5 Sharp's $8 \mathrm{~K}$ image monitor with $8 \mathrm{~K}$ rigid endoscope system. ${ }^{19}$ (A) Image of $8 \mathrm{~K}$ endoscopic surgery displayed on the screen. (B) $8 \mathrm{~K}$ image of boundary between normal cells and cancer cells. https://global.sharp/blog/2018/02/05//2/59/

\section{Conclusion}

Telemedicine services rely on ICT to deliver healthcare services and to transmit health information. This is achieved by widespread use of fast Internet, cloud computing, smartphones, computers, and mobile/connected medical devices. Video technology can effectively facilitate the growth of telemedicine. We have discussed the applications of video technology to enhance telemedicine services. The general functions of telemedicine videoconferencing systems are reviewed, and the display technology is also discussed. It is expected that advanced monitors with $8 \mathrm{~K}$ resolution can be used in telemedicine videoconferencing systems to significantly improve the quality of healthcare services, due to the advantages of lifelike images and an immersive viewing experience.

\section{Acknowledgements}

None.

\section{Conflicts of interest}

The authors declare that there is no conflict of interest.

\section{References}

1. Phaneuf A. Latest trends in medical monitoring devices and wearable health technology. 2021.

2. Majumder S, Deen MJ. Smartphone sensors for health monitoring and diagnosis. Sensors. 2019;19(9):2164.

3. WHO Global Observatory for eHealth. Telemedicine: opportunities and developments in Member States: report on the second global survey on eHealth. 2010.

4. Forbes RC, Solorzano CC, Concepcion BP. Surgical telemedicine here to stay: More support from a randomized controlled trial on postoperative surgery visits. The American Journal of Surgery. 2020;219(6):880-881.

5. Kim T, Zuckerman JE. Realizing the potential of telemedicine in global health. J Glob Health. 2019;9(2):020307.

6. Serper M, Volk ML. Current and future applications of telemedicine to optimize the delivery of care in chronic liver disease. Clinical Gastroenterology and Hepatology. 2018;16:157-161.

7. Gutkin PM, Prionas ND, Minneci MO, et al. Telemedicine in Radiation Oncology: Is It Here to Stay? Impacts on Patient Care and Resident Education. Int J Radiation Oncol Biol Phys. 2020;108(2):416-420.

8. Arora S. IoMT (Internet of Medical Things): Reducing cost while improving patient care. IEEE Pulse. 2020;11(5):24-27.

9. How Video is Shaping the Future of Telemedicine. Pexip Whitepaper

10. ECRI Institute's Healthcare Product Comparison System. Videoconferencing system, Telemedicine. 2011.

11. Ann Earon S.Transforming Healthcare with Video Communication. BlueJeans Whitepaper.

12. Barco Company. Medical imaging \& workflows.

13. Chen J, Cranton W, Fihn M. Handbook of Visual Display Technology. Dordrecht, Verlag Berlin Heidelberg. Springer; 2012.

14. Harris T. How plasma displays work. HowStuffWorks. 2002. 
15. Gurski J, Quach LM. Display Technology Overview. LYTICA White Paper; 2005.

16. Young R, O'Brien B, Tamura Y. Quantum Dots or OLEDs - Which Technology Will Dominate the TV Market of the Future? 2017.

17. Perry TS. OLED TV arrives. IEEE Spectrum. 2013;50(1):46-47.
18. Coffey VC. The age of OLED displays. Optics \& Photonics News. 2017;28(11):34-41.

19. Sharp. The Future to Be Brought by The World's First 8K Rigid Endoscope System -Introducing 8K Image Monitor into Medical Field. 2018. 